Check for updates

Cite this: RSC Adv., 2020, 10, 8703

Received 5th December 2019

Accepted 16th February 2020

DOI: $10.1039 / c 9 r a 10204 c$

rsc.li/rsc-advances

\title{
Essential oil loaded pectin/chitosan nanoparticles preparation and optimization via Box-Behnken design against MCF-7 breast cancer cell lines $\uparrow$
}

\begin{abstract}
Olivia A. Attallah, (D) ${ }^{\text {ab }}$ Amro Shetta, ${ }^{a}$ Fatma Elshishiny ${ }^{a}$ and Wael Mamdouh*a
In the continuous search for effective cancer treatments, we here report a novel anticancer nanoparticulate system composed of jasmine oil (JO), an essential oil with proven anticancer activity and pectin/chitosan composite nanoparticles (Pec/CS NPs) as encapsulating materials to overcome JO's solubility and sensitivity problems using a green ionotropic gelation method. Pec/CS/JO NPs were formulated using Box-Behnken design (BBD) to estimate the interactions and effects of studied formulation variables on particle size, zeta potential and encapsulation efficiency to develop an optimized Pec/CS nanoformulation. The nano-encapsulation system preserved the consistency of total phenolic contents in $\mathrm{JO}$ and amended its thermal stability by 1.64 fold. The antioxidant potency of JO was enhanced after encapsulation by $96.28 \%$. Consequently, the cytotoxic activity of bare Pec/CS NPs, pure JO and encapsulated $\mathrm{JO}$ in Pec/CS NPS against (MCF-7) breast cancer cells and (L-929) normal cells was evaluated using MTT assay. Encapsulated $\mathrm{JO}$ was more potent than pure $\mathrm{JO}$ with $\approx 13$ fold improvement in anticancer activity, whereas the cell viability of normal cells wasn't affected but was rather enhanced when treated with Pec/CS NPs.
\end{abstract}

\section{Introduction}

Breast cancer is one of the most common cancer types that is spread among women worldwide. ${ }^{1}$ Over the past few decades there was significant progress in cancer treatment and diagnostics. Nevertheless, killing the tumor cells selectively without harming healthy ones provides a difficult task in the field of targeted cancer therapy. ${ }^{2}$ Most of the proposed and commercially available cancer treatments are usually accompanied by side effects caused by the loss of normal cells. ${ }^{2}$ Accordingly, there is a continuous necessity to develop new tactics for nontoxic cancer treatments. ${ }^{1}$

Natural polysaccharides such as chitosan (CS) and pectin (Pec) are frequently utilized in pharmaceutical formulations due to their biodegradability and biocompatibility advantages. ${ }^{3}$ Pec is a polyanion with a large number of poly-D-galacturonic acid residues linked via $\alpha-1,4$-glycosidic bonds. ${ }^{4} \mathrm{CS}$ on the other hand is a polycation, consisting of linear chains of glucosamine and $N$-acetyl-D-glucosamine with intrinsic antimicrobial properties. ${ }^{3,5}$ Coacervation of both Pec and CS was found to mutually

\footnotetext{
${ }^{a}$ Department of Chemistry, School of Sciences and Engineering (SSE), The American University in Cairo (AUC), AUC Avenue, P.O. Box 74, New Cairo 11835, Egypt. E-mail:wael_mamdouh@aucegypt.edu

${ }^{b}$ Pharmaceutical Chemistry Department, Faculty of Pharmacy, Heliopolis University, Cairo-Belbeis Desert Road, El Salam, Cairo 11777, Egypt

† Electronic supplementary information (ESI) available. See DOI: 10.1039/c9ra10204c
}

decrease their solubility in aqueous media thus avoiding the premature release of the encapsulated agent. ${ }^{6}$ Previous research studies indicate that Pec and CS have been formulated together as beads, microparticles, nanoparticles and coacervates for the purpose of delaying or sustaining drug release. ${ }^{6-9}$ Usually, there are several physicochemical conditions that need to be observed to maintain Pec/CS nano-complexes in solution form without the development of flocculates. ${ }^{7}$ Such conditions can be handled via adequate control of the molar ratios of Pec and CS and the presence of crosslinking agent.,

We here propose the nano-encapsulation of jasmine oil (JO), an example of essential oil that is normally obtained by steam distillation of Jasminum grandiflorum (Oleaceae family) flower in Pec/CS NPs. ${ }^{10}$ JO has been used as anti-depressant, antiseptic, aphrodisiac, anti-spasmodic, expectorant, parturient and sedative. ${ }^{11-15}$ In addition, JO was found to exhibit strong cytotoxicity toward human prostate carcinoma (PC-3), human lung carcinoma (A549) and human breast cancer (MCF-7) cell lines, thus rendering it as a potential anticancer agent. ${ }^{\mathbf{1 6}}$

Unfortunately, JO as any other essential oil suffers poor solubility and has sensitivity problems which hinder its use in the various applications. Thus, the objective of this work is to improve the physical characteristics of JO and enhance its antioxidant and anticancer activities against MCF-7 breast cancer cells through its encapsulation in Pec/CS NPs. The nanoencapsulated JO (Pec/CS/JO NPs) were formulated using Box-Behnken Design (BBD) to ensure an optimum nanoformulation is produced. Pec/CS/JO NPs were prepared via 
emulsification followed by ionic gelation method as shown in (Fig. 1). Detailed experimental procedures and characterization results are given in the ESI. $\dagger$

\section{Results and discussion}

The idea of preparing Pec/CS/JO NPs by inotropic gelation of Pec was based on the inherent ability of Pec to gel in the presence of divalent cations as $\mathrm{CaCl}_{2} \cdot{ }^{17}$ Intermolecular hydrogen bonding and the electrostatic interaction between positive charge of amino groups in CS, and negative charge of carboxyl groups in Pec on the NPs' surface also served well in the cross-linking process. $^{7}$ All the above mechanisms are illustrated in (Fig. S1 $\dagger$ ). Nevertheless, to avoid the formation of outstretched chains of polymer and thus losing the nanoparticulate form, $\mathrm{NaCl}$ salt $(0.05 \mathrm{M})$ was introduced as solvent. ${ }^{17}$ Thus giving rise to a homogenous colloidal suspension. A pH 5-6 range was chosen for the fabrication process because the amino group of CS is protonated in this range. ${ }^{7}$ Pec/CS/JO NPs were obtained by mixing JO dissolved in dichloromethane with Pec solution then cross-linking the formed emulsion with a mixture of $\mathrm{CS}-\mathrm{CaCl}_{2}$ solution. BBD was used to assess the interactions and effects of formulation variables (amount of Pec, $\mathrm{CS}$, and cross-linker $\mathrm{Ca}^{2+}$ ) on three responses: particle size, zeta potential (ZP) and encapsulation efficiency (EE\%), and consequently to develop an optimized Pec/CS formulation.

Three-factor, three level BBD was created on which a series of experiments were performed. Table $\mathrm{S} 3 \dagger$ presents the matrix of the experimental runs based on the chosen independent variables and responses. The range of particle size $\left(Y_{1}\right)$ for all experiments was 468.5-698.3 nm. Similarly, ZP $\left(Y_{2}\right)$ was -18.1 to $-27.1 \mathrm{mV}$ and the $\mathrm{EE} \%\left(Y_{3}\right)$ was $24.41-28.01 \%$. A second quadratic model was applied on the studied responses and the suitability of this model was assessed by ANOVA, multiple correlation coefficient $\left(R^{2}\right)$ and lack of fit tests. The secondorder quadratic model was able to produce the highest $F$ value for the three responses $\left(Y_{1}, Y_{2}\right.$ and $\left.Y_{3}\right)$, thus, it was recognized as the fitting model. The model $P$-values, $R^{2}$, term $P$ values and lack-of-fit test for each dependent variable or response are demonstrated in Table S4. $\dagger$ In the ANOVA test, the $P$-values after applying the quadratic model on the responses $Y_{1}$, $Y_{2}$ and $Y_{3}$ were $0.0101,0.0156$ and 0.0019 , respectively. Thus,

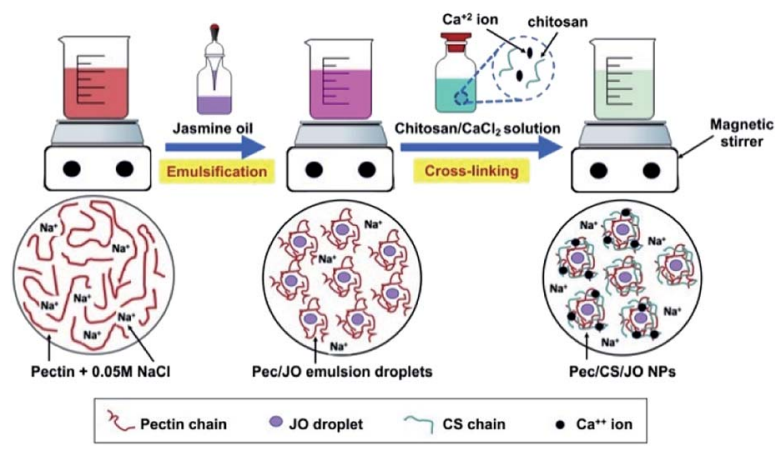

Fig. 1 Schematic presentation of JO nano-encapsulation. with a $(p<0.05)$, all of the responses fit the model well. Furthermore, the lack of fit test was applied to compare the pure error with the residual error from the replicates of design points (this study had three center points). ${ }^{18}$ A model with a nonsignificant lack of fit value (Prob. $>F$ value 0.1 ) is highly desirable. ${ }^{18}$ In the lack of fit test, the $P$-values of the quadratic model for responses $Y_{1}, Y_{2}$ and $Y_{3}$ are $0.1865,0.8424$ and 0.5970 , respectively. Thus, all of the responses presented a nonsignificant lack of fit $(p>0.1)$. Additionally, $R^{2}$ value which signifies the multiple regression analysis for the second order quadratic model were determined to measure the amount of variation around the mean proposed by the quadratic model. ${ }^{\mathbf{1 8}}$ In the current study, the adjusted $R^{2}$ values for the responses $Y_{1}$, $Y_{2}$ and $Y_{3}$ were $98.17 \%, 97.44 \%$ and $97.86 \%$, respectively.

The effects and the interactions of the studied variables on the responses were analyzed using response surface plots. Noticeably, all the studied variables affected particle size $(P$ value $<0.05)$. However, the amount of Pec $\left(X_{1}\right)$ and CS $\left(X_{2}\right)$ had a more significant effect on particle size than the amount of cross-linker; $\mathrm{CaCl}_{2}\left(X_{3}\right)$. (Fig. $\mathrm{S} 2[\mathrm{a}] \dagger$ ) shows the response surface for the relationship of particle size and amounts of Pec and CS (amount of $\mathrm{CaCl}_{2}$, fixed at $0.009 \mathrm{wt} \%$ ). At the low level of $X_{2}$, particle size increased from 468.5 to $625.2 \mathrm{~nm}$ when $X_{1}$ was increased from 0.03 to $0.07 \mathrm{wt} \%$. In addition, at the high level of $X_{2}$, the particle size increased from 548.3 to $676.6 \mathrm{~nm}$ when $X_{1}$ was increased from 0.03 to $0.07 \mathrm{wt} \%$. Thus, the rate of particle size increase was in the range of 130-150 $\mathrm{nm}$. Such results come in coordination with the work of Rampino et al. where for a given ratio between Pec and cross-linker, a size increase was observed as the mass fraction of CS increased. In addition, a remarkable size increase was observed when a high amount of Pec was used. ${ }^{4}$ The relationship between the particle size and the amount of Pec and $\mathrm{CaCl}_{2}$ is demonstrated by the response surface plot in (Fig. S2[b]†) (amount of CS, fixed at $0.025 \mathrm{wt} \%$ ). It can be observed that at low level of $X_{1}$, particle size increased from 522.5 to $552.7 \mathrm{~nm}$ when $X_{3}$ was increased from 0.006 to $0.012 \mathrm{wt} \%$. In addition, at the high level of $X_{1}$, the particle size increased from 650 to $698.3 \mathrm{~nm}$ when $X_{3}$ was increased from 0.006 to $0.012 \mathrm{wt} \%$. Thus, the increase in particle size was in the range of 25-50 nm. Pec/CS/JO NPs were prepared using Pec as the key building moiety in a medium containing CS polymer and $\mathrm{Ca}^{2+}$ ions as cross-linker. Noticeably, the amounts of CS and $\mathrm{Ca}^{2+}$ ions used in the experiments are relatively low to avoid the formation of bulk gels in the preparation of nanoparticles. ${ }^{19}$ In addition, the difference in the rate of increase of particle size at increasing ratios of $\mathrm{CS}$ and $\mathrm{Ca}^{2+}$ to Pec could indicate that the cross-links formed by $\mathrm{Ca}^{2+}$ caused a simultaneous shrink in size and an increase in compactness of the particles formed more than CS cross-links. ${ }^{3}$

$\mathrm{ZP}$ indicates the surface charge and stability of the prepared $\mathrm{Pec} / \mathrm{CS} / \mathrm{JO}$ NPs. Following Table $\mathrm{S} 4 \uparrow \mathrm{ZP}$ was significantly influenced by the amount of Pec $\left(X_{1}\right)$, the amount of CS $\left(X_{2}\right),(P$-value $<0.05)$. The amount of $\mathrm{CaCl}_{2}\left(X_{3}\right)$ did not significantly affect ZP. Pec is considered the main component of the NPs with the highest mass ratio and being anionic in nature due to the carboxylic groups the overall surface charge is expected to be negative. ${ }^{9}$ (Fig. S2[c] $\dagger$ ) shows the effects of Pec $\left(X_{1}\right)$, CS $\left(X_{2}\right)$ and 
their interactions on the $\mathrm{ZP}$ of particles $\left(Y_{2}\right)$ at the middle level of $\mathrm{CaCl}_{2}(0.009 \mathrm{wt} \%)$. The increase in the amount of Pec $\left(X_{1}\right)$ from 0.03 to $0.07 \mathrm{wt} \%$ induced an increase in the negative potential from -20.8 to $-27.1 \mathrm{mV}$ and from -19.5 to $-21.9 \mathrm{mV}$ at the lower and higher levels of CS $\left(X_{2}\right)$ respectively. Conversely, the increase in the amount of CS $\left(X_{2}\right)$ from 0.015 to $0.035 \mathrm{wt} \%$ induced a decrease in the negative potential from -20.8 to $-19.5 \mathrm{mV}$ and from -27.1 to $-21.9 \mathrm{mV}$ at the lower and higher levels of Pec $\left(X_{1}\right)$ respectively. Such decrease can be attributed to the cationic nature of CS which creates a balance between positive and negative charges on NPs surface. ${ }^{9}$

EE\% of JO $\left(Y_{3}\right)$ was significantly affected by all the independent variables $\left(X_{1}, X_{2}\right.$ and $\left.X_{3}\right)$ and the quadratic effect of $X_{1}$ $(P$-value $<0.05)$. However, the amount of Pec $\left(X_{1}\right)$ and CS $\left(X_{2}\right)$ had a more significant effect on $\mathrm{EE} \%$ than the amount of $\mathrm{CaCl}_{2}$ $\left(X_{3}\right)$ (Table S4 $\dagger$ ). Interestingly, amount of $\mathrm{CaCl}_{2}$ showed a significant interaction with amount of Pec $\left(X_{1} X_{3}\right)$ and had a positive effect on $Y_{3}$ with a coefficient of +0.15 . (Fig. S2[d] $\dagger$ ) shows the effects of $X_{1}, X_{2}$ and their interactions on JO EE\% $\left(Y_{3}\right)$ when $X_{3}$ was fixed at $(0.009 \mathrm{wt} \%)$. It can be observed that the $\mathrm{EE} \%\left(Y_{3}\right)$ was increased from 24.41 to $26.16 \%$ upon increasing the amount of $X_{1}$ from 0.03 to $0.07 \mathrm{wt} \%$ at the lower level of $X_{2}$. Similarly, at the higher level of $X_{2}$, the EE\% changed from 25.49 to $28.01 \%$ but not linearly. Such results indicate that an increase in Pec concentration led to an increase in encapsulation efficiency. This confirmed the conclusions of previous studies which elucidate that Pec has a rapid gel-forming ability and high viscosity that led to stronger hydrogel matrix and production of optimum entrapment. ${ }^{8}$ Furthermore, the coefficients of $X_{1}$ and $X_{1} X_{2}$ were positive, which means that EE\% will rise along with increasing concentration of Pec and interaction between the two polymers Pec and CS. ${ }^{8}$

The optimum formulation was then chosen based on the proposed desired criteria (minimum particle size, maximum $\mathrm{EE} \%$ and applying constrains on $\mathrm{ZP}(-20$ to $-27 \mathrm{mV}))$. The composition of optimized formulation is presented in (Table 1). A fresh batch with the optimized formula was prepared while keeping JO concentration at $2.5 \mathrm{mg}$ in order to validate the

Table 1 The optimized Pec/CS/JO NPs observed and predicted response values ${ }^{a}$

\begin{tabular}{|c|c|c|}
\hline Independent variable & & $\begin{array}{l}\text { Optimized } \\
\text { level }\end{array}$ \\
\hline$X_{1}:$ pectin conc. (wt\%) & & 0.055 \\
\hline$X_{2}:$ chitosan conc. (wt\%) & & 0.035 \\
\hline$X_{3}: \mathrm{CaCl}_{2}$ conc. $(\mathrm{wt} \%)$ & & 0.008 \\
\hline Over all desirability & & 0.714 \\
\hline Dependent variables & Expected & Observed \\
\hline$Y_{1}:$ particle size $(\mathrm{nm})$ & 604.73 & 597.7 \\
\hline$Y_{2}:$ zeta potential $(\mathrm{mV})$ & -23.04 & -23.3 \\
\hline$Y_{3}:$ encapsulation efficiency (\%) & 26.34 & 24.94 \\
\hline
\end{tabular}

calculated optimal factors and the predicted responses. Observed and predicted response values for the optimized formulation are presented in (Table 1). A fine agreement was found between the predicted and observed results which proved the validity of the suggested model. Thus, BBD succeeded in evaluating and optimizing the formulation Pec/CS/JO NPs.

After successful optimization of the Pec/CS/JO NPs, the particle size, ZP and EE\% of the prepared NPs were estimated to show the impact of varying JO concentration on the prepared NPs. As presented in (Fig. 2), JO concentration appeared to increase the particle size from $597.7 \mathrm{~nm}$ at $2.5 \mathrm{mg}$ JO to $739.4 \mathrm{~nm}$ at $10 \mathrm{mg}$ JO. The increase in the size of NPs can be attributed to the decreased coacervation between Pec and CS in the matrix due to the occupying of JO to the Pec domains. ${ }^{6}$

This was also concluded from the high $\mathrm{ZP}$ values of the formed NPs. As illustrated in (Fig. 2), Pec/CS/JO NPs had negative $\mathrm{ZP}$ values that ranged between -23.3 to $-27.2 \mathrm{mV}$. Noticeably, as the concentration of encapsulated JO increased, an increase in the zeta potential values was observed for Pec/CS/JO NPs.

Furthermore, the effect of added JO amounts on $\mathrm{EE} \%$ of Pec/ CS/JO NPs is demonstrated in (Fig. 2). It can be noticed that as the concentration of JO increased from $2.5 \mathrm{mg}$ to $10.0 \mathrm{mg}$ the $\mathrm{EE} \%$ was increased from $8.60 \%$ to $30.30 \%$. Such low EE\% can be attributed to the limited physical interactions between the hydrophobic JO and the hydrophilic polymer matrix where the majority of JO is likely to be localized on the surface of the particles. ${ }^{20}$ In such case, JO molecules on the particle surface were easily washed away during the washing and centrifugation processes, thus resulted in reduced EE. Another reason for low $\mathrm{EE} \%$ against theoretical JO loading could be the decrease in NPs recovery which led to an enhanced JO loss. ${ }^{20}$

Moreover, the loading capacity\% (LC\%) of encapsulated JO was determined according to the procedures mentioned in (ESI†). As shown in (Fig. 3), the values ranged from 0.43-6.06\%. Such results indicated that initial JO content had a significant effect on LC\% which reached its maximum at $10 \mathrm{mg}$

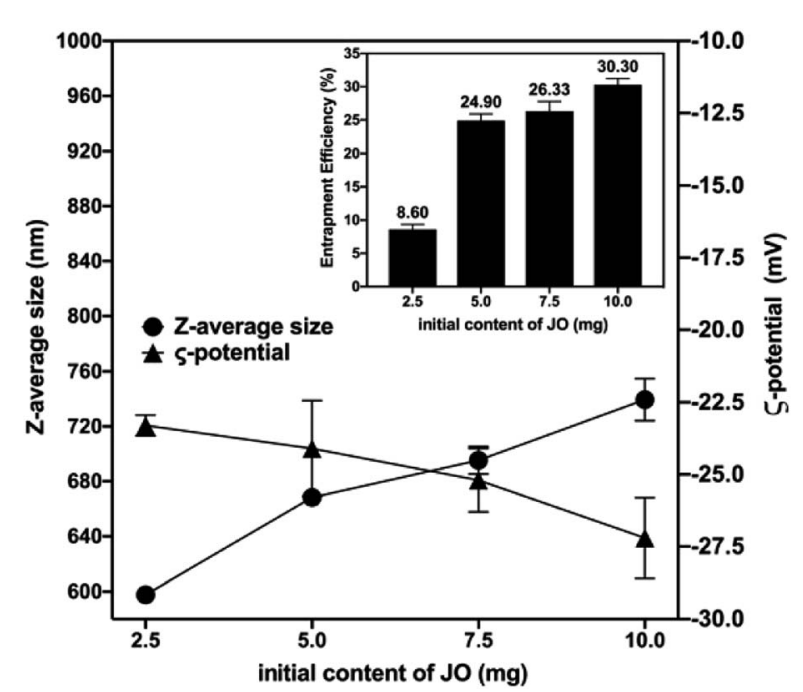

Fig. 2 Effect of initial content of JO on particle size $(\mathrm{nm})$, zeta potential $(\mathrm{mV})$ and encapsulation efficiency (EE\%). 


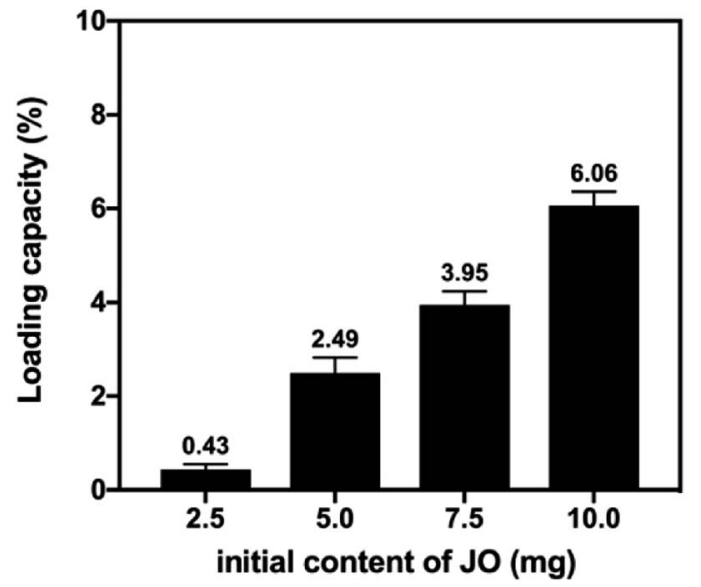

Fig. 3 Effect of initial content of JO on loading capacity (LC\%).

concentration of JO. Thus, $10 \mathrm{mg}$ concentration for JO was selected to be the optimum content of JO for the rest of the study.

Different characterization techniques were performed to determine the physical and crystalline properties of Pec/CS/JO NPs. TEM images (Fig. S3†) of Pec/CS/JO NPs revealed spherical NPs with an average size of $150 \pm 4 \mathrm{~nm}$ while the XRD pattern (Fig. S5 $\dagger$ ) showed a broad typical hump of amorphous material with the absence of the characteristic crystalline Pec peaks, indicating that Pec was changed to a noncrystalline form during the preparation process. Thermogravimetric analysis (TGA) was also executed to study the thermal stability of JO, Pec/ CS NPs and Pec/CS/JO NPs. The temperature of maximum degradation rate $\left(T_{\mathrm{d}}\right)$ for JO, Pec/CS NPs and Pec/CS/JO NPs was estimated from the first derivative of TGA curve at the maximum slopes of weight change (DTG) (Fig. 4[b]). TGA thermogram (Fig. 4[a]) of JO exhibited one-step mass loss starting at $50{ }^{\circ} \mathrm{C}$ and the value of $T_{\mathrm{d}}$ for pure JO was $154{ }^{\circ} \mathrm{C}$. Accordingly, these results designate the low thermal stability of pure JO. On the other hand, Pec/CS NPs showed two-step mass loss with $T_{\mathrm{d}}$ values of $65{ }^{\circ} \mathrm{C}$ and $241{ }^{\circ} \mathrm{C}$. Encapsulated JO in Pec/CS NPs exhibited a slight increase in the second step $T_{\mathrm{d}}$ value reaching $248{ }^{\circ} \mathrm{C}$ as indicated in (Fig. $4[\mathrm{~b}]$ ). Such observation designates an improvement in encapsulated JO's thermal stability by about
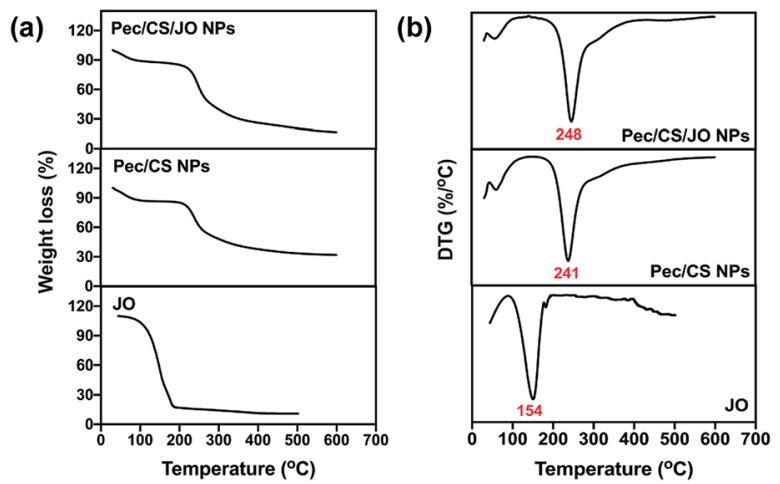

Fig. 4 TGA (a), and DTG (b) spectra of pure JO, Pec/CS NPs, and Pec/ CS/JO NPS.
1.64 folds. Thus, Pec/CS NPs presented an acceptable thermal sheath for the encapsulation of JO.

In vitro release study of JO from $\mathrm{Pec} / \mathrm{CS} / \mathrm{JO}$ NPs was performed in different $\mathrm{pH}$ buffers (3.0, 5.5, and 7.4) for $48 \mathrm{~h}$ (Fig. 5). Noticeably, the medium $\mathrm{pH}$ had a great impact on JO release. JO released faster at $\mathrm{pH} 3.0$ than at $\mathrm{pH} 5.5$ and 7.4. At $\mathrm{pH} 3.0$, both JO and Pec/CS NPs are positively charged, and the electronic repulsion facilitates the diffusion of JO from the NPs to the liquid phase. ${ }^{7}$ At $\mathrm{pH} 5.5$ (pH used during crosslinking process), both JO and Pec/CS NPs were more hydrophobic, and the electrostatic interactions between them may cause low release of JO through the diffusion effect. Thus pH 5.5 showed the slowest release paradigm. At $\mathrm{pH} 7.4 \mathrm{JO}$ release percentage was quite high in comparison to that of $\mathrm{pH} 5.5$, possibly because of the swelling of the NPs and their erosion at this $\mathrm{pH}^{8}{ }^{8}$ In vitro release of JO was also tested against different release kinetics (Table S5†).

The release of JO from the Pec/CS/JO system followed Korsmeyer-Peppas model $\left(R^{2}\right.$ above 0.76$)$ which is based on plotting the log values of cumulative release percentage of JO against log time as shown in (Fig. S7 $\dagger$ ). Following Korsmeyer-Peppas model analysis, the release of the JO from Pec/CS/JO system is based on the diffusion from Pec/CS/JO matrix where the diffusion exponential $(n)$ showed values below 0.45 in all buffers used. ${ }^{21}$ The diffusion constant $(k)$ value was also used to study the release rate. The value of $k$ was the highest for the release in acetate buffer solution with $\mathrm{pH}$ of $3.0\left(39.26 \mathrm{~min}^{-1}\right)$ indicating the fastest release rate among the other buffers used whereas the acetate buffer solution with $\mathrm{pH}$ of 5.5 showed the lowest $K$ value $\left(21.74 \mathrm{~min}^{-1}\right)$ indicating the slowest release rate.

Total phenolic content (TPC) of pure JO, Pec/CS NPs, and encapsulated JO in Pec/CS NPs was quantified using FolinCiocalteu reagent. ${ }^{22}$ As displayed in (Fig. 6), each gram of bare Pec/CS NPs, pure JO and encapsulated JO in Pec/CS NPs was found to be equivalent to $81.97,1116.11$ and $1823.45 \mathrm{mg} \mathrm{GAE}$, respectively. Noticeably, pure and encapsulated JO possessed higher TPC than Pec/CS NPs. Nevertheless, the TPC identified for encapsulated JO was significantly higher than that of the

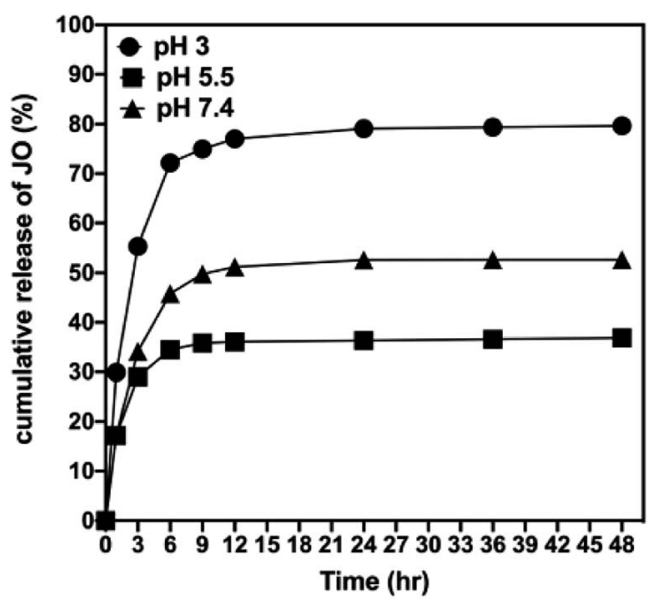

Fig. 5 In vitro release profiles of $\mathrm{JO}$ from Pec/CS NPs in different $\mathrm{pH}$ media: $\mathrm{pH} 3,5.5$, and $\mathrm{pH} 7.4$. 


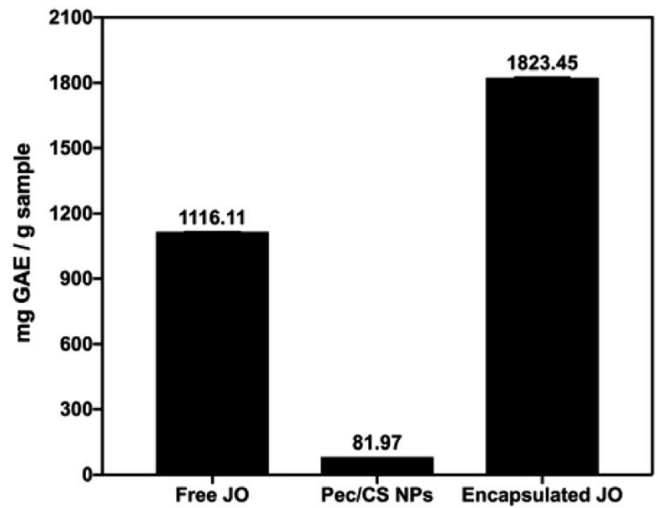

Fig. 6 TPC of pure JO, Pec/CS NPs, and encapsulated JO in Pec/CS NPs expressed in (mg GAE/g sample).

free JO (by 63.38\%). Such results can be attributed to the increase in the surface area owing to oil droplet size reduction. ${ }^{22}$

The antioxidant activity of encapsulated JO in Pec/CS NPs was also evaluated using 2,2-diphenyl-1-picrylhydrazyl radical (DPPH) free radical scavenging assay to determine the effect of nano-encapsulation on the oxidative properties of JO. ${ }^{22,23}$ Gallic acid which is a powerful antioxidant was used as a positive control with $\mathrm{IC}_{50}$ of $0.078 \mathrm{mg} \mathrm{mL} \mathrm{m}^{-1} \cdot{ }^{22}$ As shown in (Fig. 7) Pec/ CS NPs displayed an acceptable antioxidant activity with $\mathrm{IC}_{50}$ of $0.385 \mathrm{mg} \mathrm{mL}^{-1}$. This can be attributed to the DPPH scavenging activity of pectin, where the hydroxyl groups and electron transfer from ( $\mathrm{ROH}$ or $\mathrm{RO}-$ ) of pectin to $\mathrm{DPPH}^{*}$ play an important role in termination of the radical chain reaction. ${ }^{23}$ On the other hand, pure JO and encapsulated JO in Pec/CS NPs had

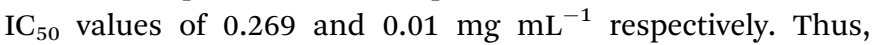
encapsulated JO antioxidant activity was significantly higher than that of pure JO (by $96.28 \%$ ) which might be due to the combined antioxidant activity of both Pec and JO giving rise to more reactive oxygen species scavenging properties. ${ }^{23}$

Consequently, the cytotoxicity of JO before and after encapsulation in Pec/CS NPs was determined on MCF-7 cell lines (ATCC HTB-22 ${ }^{\mathrm{TM}}$ ) as an assessment of anticancer activity and on normal L-929 cell lines (ATCC CCL-1 ${ }^{\mathrm{TM}}$ ) to evaluate the biocompatibility of the proposed nano-formulation. Both cell

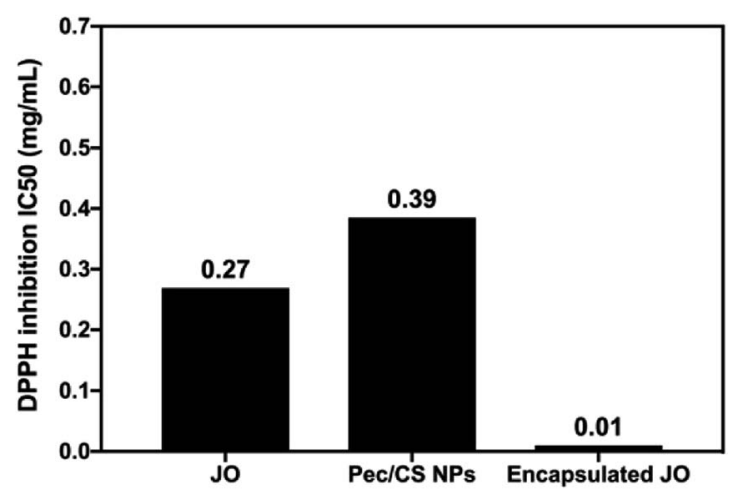

Fig. $7 \quad I_{50}$ values for DPPH radical scavenging assay of pure $\mathrm{JO}, \mathrm{Pec} /$ CS NPs, and encapsulated JO in Pec/CS NPs. lines were purchased from the American Type Culture Collection (ATCC). The percent viability of MCF-7 and L-929 cells obtained for bare Pec/CS NPs (zero JO concentration), pure JO and encapsulated JO in Pec/CS NPs are shown in Fig. 8[a], [b] and [c] respectively. The obtained results revealed that the cytotoxicity of pure and encapsulated JO was concentration dependent and there was a decrease in the viability of the MCF7 cells as the concentration of JO increased. On the other hand, almost improved cell viability was observed for the fibroblast cells (L-929 cells) when treated with bare Pec/CS NPs, pure JO and encapsulated JO in Pec/CS NPs. This indicates that the L929 were metabolically active in the presence of the proposed nano-formulation which validate its biocompatibility. Such results come in coordination with reported literature on the efficacy of pectin and chitosan formulated as scaffolds for enhanced proliferation of L-929 cells ${ }^{24,25}$

Moreover, the $\mathrm{IC}_{50}$ values were calculated for MCF-7 cells and were found to be $3.17,0.32$ and $0.0246 \mathrm{mg} \mathrm{mL}^{-1}$ after $24 \mathrm{~h}$ treatment with bare Pec/CS NPs, pure JO and encapsulated JO in $\mathrm{Pec} / \mathrm{CS}$ NPs, respectively. The $\mathrm{IC}_{50}$ values decreased $\approx 13$ fold for encapsulated JO than the pure JO (Fig. 9). Such results can be
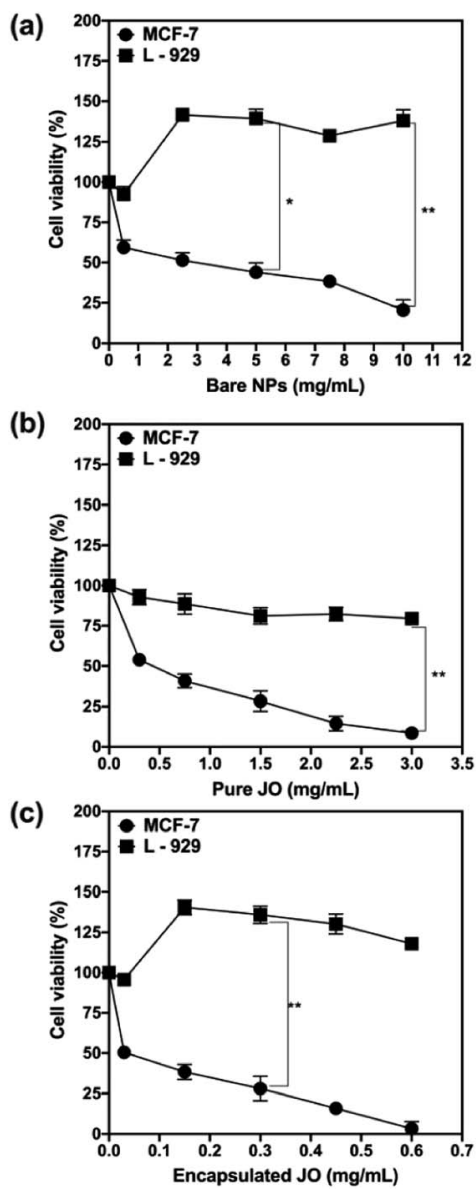

Fig. 8 The percentage of viable MCF-7 and L-929 cells as estimated by the MTT assay after $24 \mathrm{~h}$ incubation with: (a) bare NPs, (b) pure JO and (c) encapsulated $\mathrm{JO}$ in Pec/CS NPs. The data are displayed as average of three independent experiments (mean \pm SD). Significance of $P$-values between the two cell lines were denoted by $(* P<0.05$ and $* * P<0.01)$ 


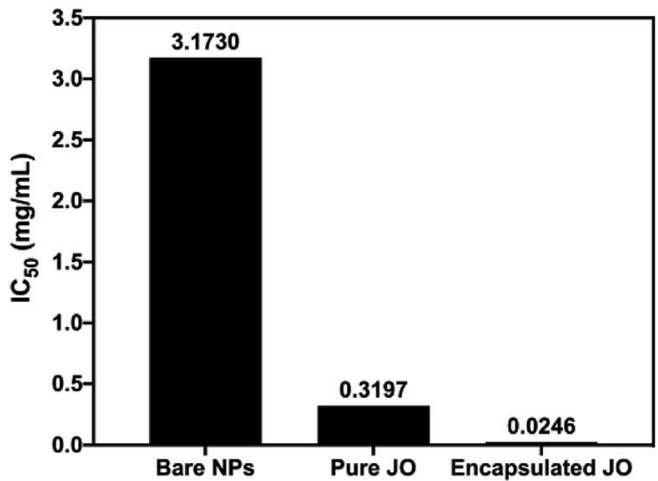

Fig. 9 Estimated $\mathrm{IC}_{50}$ values of bare NPs, pure $\mathrm{JO}$ and encapsulated $\mathrm{JO}$ in Pec/CS NPs against MCF-7 cells.

attributed to the role played by the proposed nano-particulate system (Pec/CS) in enhancing the cytotoxic effect of JO, since earlier studies indicated that pectin induces DNA fragmentation which leads to cellular apoptosis in cancer cells. ${ }^{26}$

\section{Conclusions}

The present study successfully demonstrated that JO can be encapsulated by Pec/CS polyelectrolyte complex cross-linked with $\mathrm{Ca}^{2+}$. The effects of the three independent variables (amount of Pec, amount of $\mathrm{CS}$ and amount of $\mathrm{CaCl}_{2}$ ) on particle size, ZP and EE\% were evaluated by BBD to study effects of the formulation variables on the studied responses. In addition, the results of the optimized formulation were very close to the estimated values, suggesting the success of the BBD in formulating the nanoparticulate system. In vitro release experiments demonstrated that JO was released from Pec/CS NPs faster at $\mathrm{pH}$ 3.0 than at pH 5.5 and $\mathrm{pH} 7.4$ and the release tended to follow Korsmeyer-peppas model. TGA and Folin-Ciocalteu assay results showed the ability of Pec/CS NPs system to preserve the thermal stability and TPC of JO respectively. Encapsulated JO also exhibited excellent cyto-compatibility against normal cells and demonstrated better antioxidant and anticancer properties than free JO. Thus, we can conclude that nanoencapsulation is a useful tool for stabilizing the physicochemical properties of EOs like JO and improve their effectiveness for the various applications.

\section{Conflicts of interest}

There are no conflicts to declare.

\section{Acknowledgements}

The authors acknowledge the financial support received from the American University in Cairo (AUC) through Faculty Support Research Grants.

\section{Notes and references}

1 N. M. Elbaz, L. Ziko, R. Siam and W. Mamdouh, Sci. Rep., 2016, 6, 30729.

2 K. Das, S. Nimushakavi, A. Chaudhuri and P. K. Das, ChemMedChem, 2017, 12, 738-750.

3 S. Pistone, F. M. Goycoolea, A. Young, G. Smistad and M. Hiorth, Eur. J. Pharm. Sci., 2017, 96, 381-389.

4 A. Rampino, M. Borgogna, B. Bellich, P. Blasi, F. Virgilio and A. Cesàro, Eur. J. Pharm. Sci., 2016, 84, 37-45.

5 N. P. Birch and J. D. Schiffman, Langmuir, 2014, 30, 34413447.

6 S. O. Syed Mohamad Al-Azi, Y. T. F. Tan and T. W. Wong, React. Funct. Polym., 2014, 84, 45-52.

$7 \mathrm{H}$. Wang, American Journal of Polymer Science and Technology, 2018, 3, 82 .

8 A. Fahrurroji, D. Thendriani and H. Riza, Int. J. Pharmacol. Pharm. Sci., 2017, 9, 98.

9 P. Ahlin Grabnar and J. Kristl, Pharmazie, 2010, 65, 851-852.

10 O. Prakash, D. Sahoo and P. K. Rout, Nat. Prod. Commun., 2012, 7, 89-92.

11 E. E. Elisha, S. J. Al-Maliki and D. K. Ibrahem, Pharm. Biol., 1988, 26, 221-227.

12 M. H. Hur, Y. S. Yang and M. S. Lee, J. Evidence-Based Complementary Altern. Med., 2008, 5, 325-328.

13 G. Zhao, Z. Yin and J. Dong, J. Ethnopharmacol., 2009, 125, 265-268.

14 T. Hongratanaworakit, Nat. Prod. Commun., 2010, 5, 157162.

15 C. Rath, S. Devi, S. Dash and R. Mishra, Indian J. Pharm. Sci., 2009, 70, 238.

16 Y. Zu, H. Yu, L. Liang, Y. Fu, T. Efferth, X. Liu and N. Wu, Molecules, 2010, 15, 3200-3210.

17 H. Jonassen, A. Treves, A. L. Kjøniksen, G. Smistad and M. Hiorth, Biomacromolecules, 2013, 14, 3523-3531.

18 D. W. Lee, N. Marasini, B. K. Poudel, J. H. Kim, H. J. Cho, B. K. Moon, H. G. Choi, C. S. Yong and J. O. Kim, J. Microencapsulation, 2014, 31, 31-40.

19 C. Y. Yu, Y. M. Wang, N. M. Li, G. S. Liu, S. Yang, G. T. Tang, D. X. He, X. W. Tan and H. Wei, Mol. Pharm., 2014, 11, 638644.

20 I. Tekko, A. Maaz, W. Abdelwahed and S. Trefi, International Journal of Academic and Scientific Research, 2015, 3, 1-12.

21 P. L. Ritger and N. A. Peppas, J. Controlled Release, 1987, 5, $37-42$.

22 A. Shetta, J. Kegere and W. Mamdouh, Int. J. Biol. Macromol., 2019, 126, 731-742.

23 S. Liu, X. Shi, L. Xu and Y. Yi, Chin. J. Oceanol. Limnol., 2015, 34, 372-381.

24 N. zhao Yao, C. Huang and D. di Jin, Orthop. Surg., 2009, 1, 231-237.

25 H. Y. Lin, H. H. Chen, S. H. Chang and T. S. Ni, J. Biomater. Sci., Polym. Ed., 2013, 24, 470-484.

26 S. K. Niture and L. Refai, Am. J. Pharmacol. Toxicol., 2013, 8, 9-19. 